\title{
An Improved Watershed Segmentation Algorithm for Adhesive Particles in Sugar Cane Crystallization
}

\author{
Yanmei Meng ${ }^{a}$, Kangyuan Zheng $^{b}$, Feihong $\mathrm{Hu}^{\mathrm{b}}$, Wenxing $\mathrm{Li}^{\mathrm{b}}$, Guancheng \\ $\mathrm{Lu}^{\mathrm{b}}$ \\ College of Mechanical Engineering, Guangxi University, Nanning 530000, China \\ agxu_mengyun@163.com, bomkey04@163.com
}

Keywords: Adhesive particles, Watershed, Image segmentation, Distance transformation, Area threshold, Shape factor

\begin{abstract}
Cane sugar crystallization, which has both heat and mass transfer, is a very complex chemical and physical process. Due to the constraints of technical conditions, detection of crystals is mainly relied on artificial methods with disadvantages of low accuracy and subjective judgment errors. Application of image processing technology can achieve obtaining the particle information of crystals automatically. However, adhesive phenomenon of collected image is serious, which causes difficulties for the subsequent image recognition. According to the problem of difficulties in segmenting adhesive particles for sugar cane crystallization, an improved watershed segmentation algorithm is proposed in this paper. Adhesive particles are remained by using area threshold and shape factor, and the single-completed particles are removed temporarily. The seed points are optimized by distance transformation, thus obtaining the simplified particle image. By using watershed algorithm to segment the simplified particle image and overlapping the single-completed particles with segmented result, the final image segmentation result is obtained. Experimental results show that the improved watershed algorithm can eliminate the irregular details, area noise and isolated edge pixels effectively.
\end{abstract}

\section{Introduction}

Using image processing technology in the detection of crystals in sugar crystallization process, will help to realize real-time decision control. Because of the great viscosity between particles, the collected sugar crystallization particles image will appear mutual adhesion and overlapping phenomenon, which is unfavorable for subsequent feature extraction and related measurement [1-4]. Watershed algorithm is a classical image segmentation algorithm based on region segmentation. However, due to the influence of noise and regional details, traditional segmentation algorithm has many local minimal values, which causes serious over-segmentation easily [5]. Many scholars have proposed algorithms to solve over-segmentation problems, but all have much limitation. Bieniceki uses median filtering and Gaussian filtering to solve over-segmentation problems, but the edge information is usually kept poorly because above filtering techniques remove high frequency parts [6]. Y. R. You proposes a watershed segmentation algorithm for adhesive particles combined with distance transformation [7]. Hairs proposes a watershed segmentation algorithm to alleviate over-segmentation phenomenon based on region merging. But this algorithm causes too much small region, which influences its running speed because of large workload [8]. From above, image segmentation based on watershed has caused many research interests, but the problem of over-segmentation existing in adhesive particles is not solved well.

According to the difficulties in segmenting adhesive particles for sugar cane crystallization, an improved watershed segmentation algorithm is proposed in this paper. Two parameters, which are shape factor and area threshold, is used to choose adhesive particles, and the single-completed particles are removed temporarily. The seed points are optimized by distance transformation, thus obtaining the simplified particle image. By using watershed algorithm to segment the simplified particle image and overlapping the single-completed particles with segmented result, the final image segmentation result is obtained. Experimental results show that the improved watershed 
algorithm can eliminate the irregular details, area noise and isolated edge pixels effectively. And the crystal image with mutual adhesion can be segmented satisfactorily by this improved algorithm.

\section{Watershed Algorithm}

\subsection{Watershed algorithm and its mathematical description}

Watershed algorithm is a classical algorithm based on mathematical morphology and region segmentation. Main goal of watershed algorithm is to identify the watershed. Supposed that there is hole in the position of each region's minimum, water would rise from the hole at a uniform rate. When the water in different basins is aggregated, the dam will prevent them from polymerization. In other words, the water can only reach the top of the dam, and the boundaries correspond to the dividing lines of watershed. And the dividing lines are extracted from watershed algorithm [9].

\subsection{Watershed segmentation algorithm based on distance transformation for sugar cane crystals}

According to the morphology of the sugar cane crystals, distance transformation is carried out in the crystal particles image based on hole filling. Distance transformation is a method that can calculate the minimum distance from background to each pixel. Generally, Euclidean distance transformation is chosen to create gray gradient image. Although this method can segment adhesive particles, there are also much over-segmentation phenomenon. Segmentation results are shown as Fig. 1.

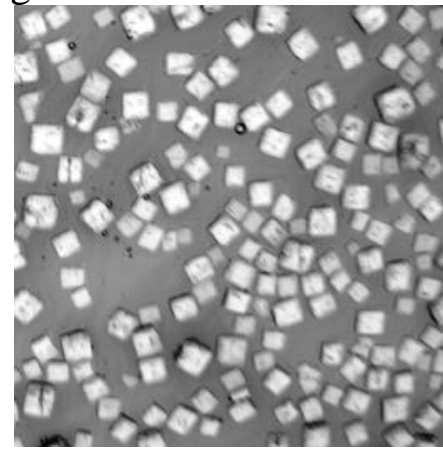

(a) Original image

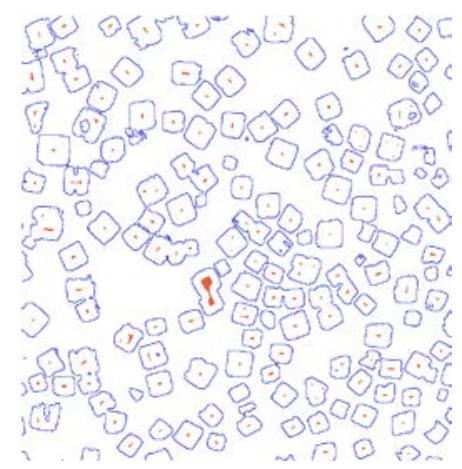

(b) Seeds image after distance transformation

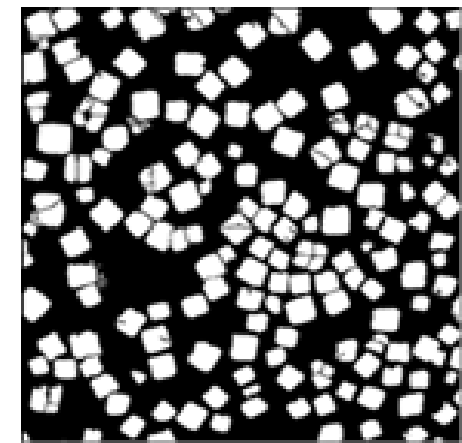

(c) Watershed segmentation results

Fig. 1. Watershed segmentation results of adhesive sugar cane crystals image based on distance transformation

\subsection{Watershed algorithm based on local minimal values}

Because some crystal particles have irregular shape, there are more than one seed in some area. According to the principle of watershed segmentation, one segmentation region will generate if there exists one local minimal value [10]. Therefore, if there exists many local minimal values in the crystal particle, over-segmentation will happen. The seed points belonging to the same particle should be merged into a region after seed points searching. Every two seed points are checked. As is shown in Eqs. (1) (3), by judging whether their distance is bigger than 1.2 times of the bigger distance, the one which is smaller will be remained. Among them, $k_{1}, k_{2}$ represent two seed points of the same local area, and $k_{3}$ is a point of the boundary. $k_{1} k_{3}, k_{2} k_{3}$ represent the distance between two seed points and the boundary point.

$$
\begin{gathered}
E=M A X\left(k_{1} k_{3}, k_{2} k_{3}\right) \\
f k_{1} k_{2}<1.2 E \text { then } T=\text { true } \\
\text { else } T=\text { false }
\end{gathered}
$$

If $T$ is true, these two seed points are in the same region. Otherwise they belong to different regions. 


\section{Improved Watershed Algorithm}

According to the problem of over-segmentation existed in traditional watershed algorithm, an improved watershed algorithm is proposed by this paper. Shape factor and area threshold are used to choose adhesive particles, and the single-completed particles are removed temporarily, which lays foundation to reduce over-segmentation. The whole improved watershed segmentation algorithm is shown as Fig. 2.

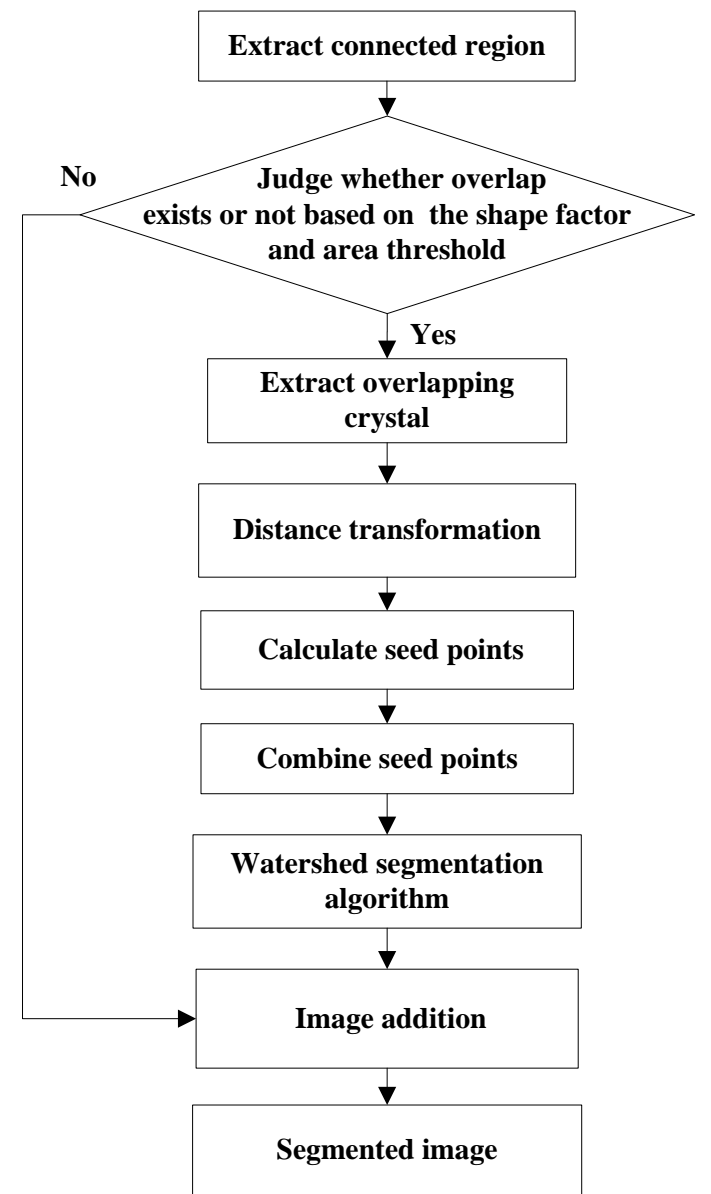

Fig. 2. Improved watershed segmentation algorithm

Chain code tracking method is taken to calculate the area and perimeter of twenty groups of adhesive particles and single-completed particles, and the shape factor is calculated as well. Sugar crystal area $S_{i}$ is acquired through the line table, and the region area is acquired by cumulating length of the line, where $X_{j R}$ and $X_{j L}$ are the right point and left point of $j-t h$ line. Sugar crystal perimeter $L_{i}$ is acquired by calculating eight-connected chain code, where $N_{i 1}$ and $N_{i 2}$ are the pixel number of the horizontal and vertical direction, and $N_{i 3}$ is the pixel number of $3 \times 3$ area in the diagonal direction. The shape factor is calculated by $\mathrm{Eq} P=L^{2} / 4 \pi S$. The final results are shown in Table 1. 
Table. 1. Characteristic parameter results of sugar cane crystals

\begin{tabular}{crrr|rrrr}
\hline \multicolumn{4}{c}{ Adhesive particles } & \multicolumn{4}{c}{ Non-adhesive particles } \\
No. & Area $/ \mathrm{mm}^{2}$ & Piemeter/mm & Shape factor & No. & Area $/ \mathrm{mm}^{2}$ & Piemeter/mm & Shape factor \\
\hline 1 & 0.411 & 2.792 & 0.663 & 1 & 0.206 & 1.420 & 1.286 \\
2 & 0.247 & 1.858 & 0.899 & 2 & 0.176 & 1.334 & 1.241 \\
3 & 0.336 & 2.382 & 0.745 & 3 & 0.240 & 1.677 & 1.072 \\
4 & 0.452 & 2.706 & 0.775 & 4 & 0.233 & 1.534 & 1.244 \\
5 & 0.651 & 3.820 & 0.560 & 5 & 0.235 & 1.505 & 1.300 \\
6 & 0.507 & 3.001 & 0.707 & 6 & 0.236 & 1.505 & 1.309 \\
7 & 0.414 & 2.792 & 0.668 & 7 & 0.210 & 1.648 & 0.971 \\
8 & 0.712 & 4.202 & 0.507 & 8 & 0.192 & 1.381 & 1.265 \\
9 & 0.569 & 3.430 & 0.607 & 9 & 0.209 & 1.458 & 1.234 \\
10 & 0.466 & 3.411 & 0.504 & 10 & 0.236 & 1.515 & 1.292 \\
11 & 1.229 & 7.012 & 0.314 & 11 & 0.162 & 1.277 & 1.251 \\
12 & 0.378 & 2.668 & 0.667 & 12 & 0.197 & 1.381 & 1.296 \\
13 & 0.479 & 2.792 & 0.772 & 13 & 0.211 & 1.448 & 1.264 \\
14 & 0.294 & 2.048 & 0.879 & 14 & 0.208 & 1.496 & 1.165 \\
15 & 0.295 & 2.458 & 0.614 & 15 & 0.208 & 1.496 & 1.165 \\
16 & 0.332 & 2.382 & 0.735 & 16 & 0.203 & 1.391 & 1.319 \\
17 & 0.293 & 1.953 & 0.967 & 17 & 0.211 & 1.486 & 1.202 \\
18 & 0.415 & 2.439 & 0.876 & 18 & 0.220 & 1.648 & 1.016 \\
19 & 0.332 & 2.382 & 0.735 & 19 & 0.203 & 1.477 & 1.169 \\
20 & 0.411 & 2.792 & 0.663 & 20 & 0.206 & 1.420 & 1.286 \\
\hline
\end{tabular}

From Table 1, the critical values of sugar crystal particles can be obtained with shape factor $f=0.9$ and mean area $S_{V} \times 0.7$. If the shape factor $f<0.9$ and mean area $S>S_{V} \times 0.7$, these particles are adhesive. Otherwise they are single-completed. The judging laws are shown as Eqs. (4) (6).

$$
\begin{aligned}
& \text { if } \quad\left(S>0.7 \times S_{V}\right) \& \& f<0.9 \\
& \text { then } K=\text { true } \\
& \text { else } K=\text { false }
\end{aligned}
$$

If $K$ is true, these particles are adhesive. Otherwise they are single-completed.

\section{Experimental Results and Analysis}

In order to prove the validity of this improved algorithm, a group of crystal particle images with adhesive phenomenon is collected by this paper. And this algorithm is tested in Matlab environment. After using image preprocessing algorithms, such as linear gray transformation and binarization, the improved watershed segmentation algorithm is run to segment adhesive particle image. Experimental results are shown as Fig. 3. Among them, Fig. 3(a) is the original image of crystal particles, and Fig. 3(b) is the extracted adhesive particles. Fig. 3(c) is the seeds image after distance transformation, and Fig. 3(d) is the same local seeds merging image. Fig. 3(e) is the segmentation image of adhesive particles, and Fig. 3(f) is the overlapping image of segmentation result and single-completed particles. By combining area threshold and shape factor to separate the single-completed particles, and taking watershed algorithm to segment the image based on distance transformation, the adhesive crystal particles is separated reasonably. 


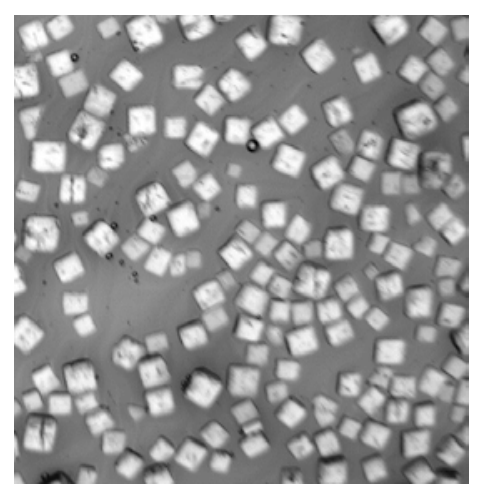

(a) Original image

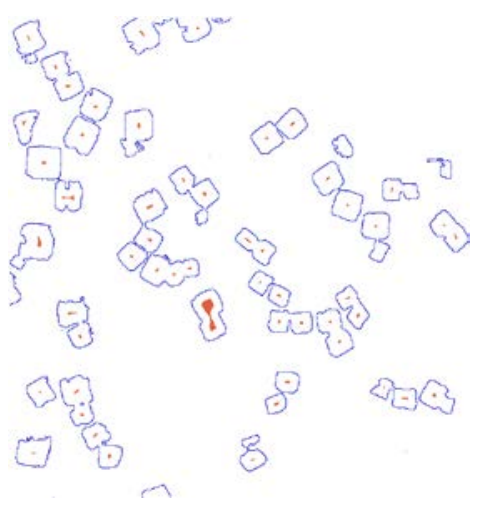

(d) Same local seeds merging image

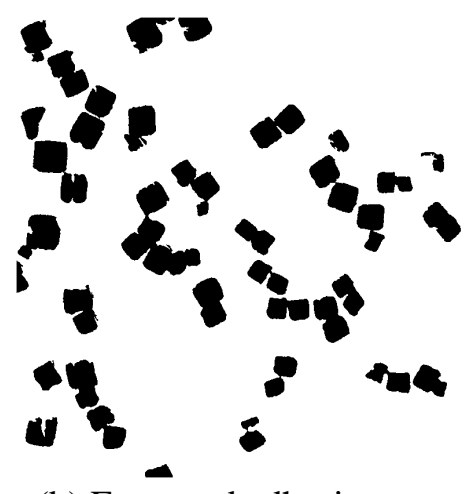

(b) Extracted adhesive

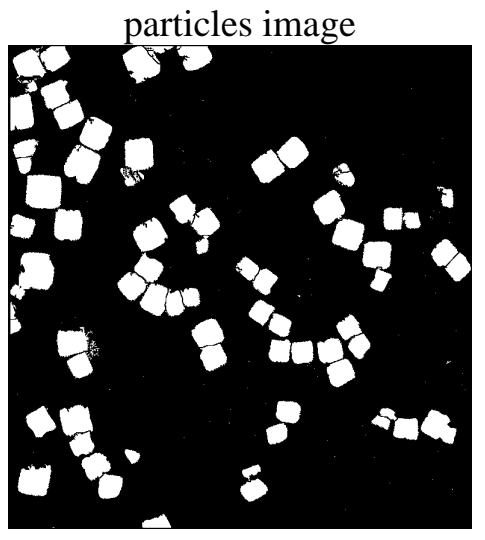

(e) Watershed segmentation image

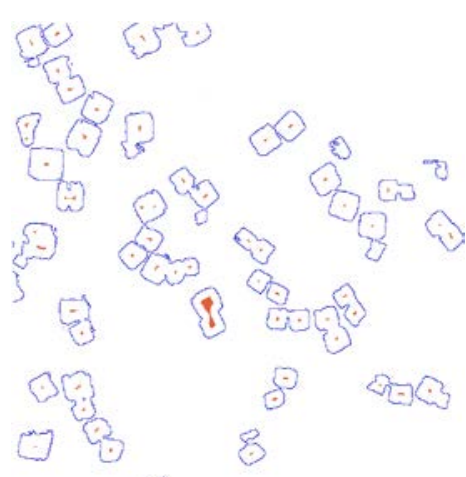

(c) Seeds image after distance transformation

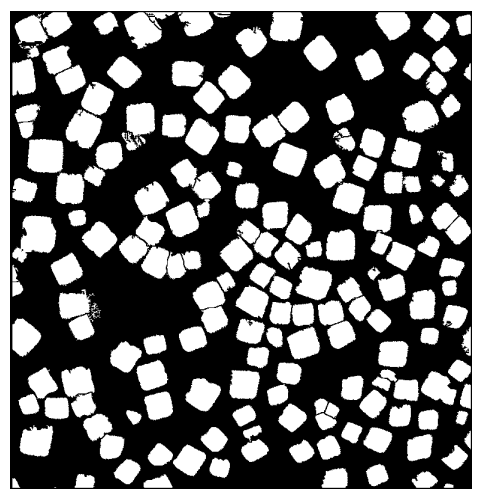

(f) Overlapping image of segmentation result and single-completed particles

Fig. 3. Results of improved watershed algorithm

In order to better analyze the experimental results, two evaluation parameters are defined as Eqs. (7) and (8), and especially, the performance of segmentation result is decided by subjective visual evaluation.

$$
\begin{aligned}
R_{1} & =N_{1} / N \\
R_{2} & =S_{1} / S
\end{aligned}
$$

$R_{1}$ is the over-segmentation rate. $N_{1}$ is the number of over-segmented particles by this proposed algorithm. $N$ is the total number of particles. $R_{2}$ is the removal rate. $S_{1}$ is the number of removed adhesive particles actually by this improved algorithm. $S$ is the number of particles that should be removed by subjective judgment.

The experimental results are shown as Table 2. It can be seen that this improved algorithm has a remarkable result in reducing over-segmentation rate and increasing removal rate. What's more, this proposed algorithm can eliminate over-segmentation phenomenon for different morphological particles.

Table 2. Compared results

\begin{tabular}{cccc}
\hline & Traditional watershed algorithm & Improved watershed algorithm \\
\hline Over-segmentation rate & $R_{1}$ & $37.3 \%$ & $3.5 \%$ \\
Removal rate $R_{2}$ & $78.9 \%$ & $92.2 \%$ \\
\hline
\end{tabular}

\section{Conclusion}

According to the over-segmentation problems existing in the adhesive particles image, an improved algorithm is proposed based on distance transformation and two parameters, which are shape factor and area threshold. These two parameters are used to choose adhesive particles, and the single-completed particles are removed temporarily. The seed points are optimized by distance 
transformation, thus obtaining the simplified particle image. By using watershed algorithm to segment the simplified particle image and overlapping the single-completed particles with segmented result, the final image segmentation result is obtained. Compared with traditional watershed, this improved algorithm reduces over-segmentation and is more suitable to segment adhesive crystal particles image. Experimental results show that the improved watershed algorithm can eliminate the irregular details, area noise and isolated edge pixels effectively.

\section{Acknowledgement}

This work is supported by National Natural Science Foundation of China (No. 51465003), Innovation Project of Guangxi Graduate Education (No. YCSZ2014029), Scientific and Technological Project of Nanning City (No. 20131079), and Guangxi Manufacturing System and Advanced Manufacturing Technology Laboratory Project (No. 14-045-15S08).

\section{References}

[1] JP. Thiran, Automatic recognition of cancerous cells using mathematical morphology, In: Proc of Int Conf on SPIE Rochester. 2359(1994) 392-401.

[2] KS. Kim, J. Song, F. Golshani, Automatic classification of cell using morphological shape in Peripheral blood images, n: Proc of Int Conf on SPIE Boston. 4210(2000) 290-298.

[3] V. D. Berg E H, A. G. C. A Meesters, J. A. M. Kenter, et.al, Automated separation of touching grains in digital images of thin sections, Computers \& Geosciences. 28(2002) 179-190.

[4] D. Zhang, Z. Xie, J. S. Ai, Research on rebar section positioning method in online rebar counting system, Chinese Journal of Scientific Instrument. 31(2010) 1173-1178.

[5] JBTM. Roerdink, A. Meijster, The watershed transform: Definitions, algorithms and parallelization strategies, Fundamental informaticae, IOS Press: 357-364, 2000.

[6] W. Bieniecki, Over segmentation avoidance in watershed-based algorithms for color images, Proceedings of the International Conference, Lodz, Poland: [s. n.], 2004: 169-172.

[7] Y. R. You, Y. L. Fan, Q. Pang, Separate Algorithm for Overlapping Cell Images Based on Distance Transformation, Computer Engineering and Application. 41(2005) 207-208.

[8] K. Haris, SN. Efstratiadis, N. Maglaveras, Hybrid image segmentation using watersheds and fast region merging, IEEE Trans on Image Processing. 7(1998) 1684-1698.

[9] L. Vincent, P. Soille, Watersheds in digital spaces: an efficient algorithm based on immersion simulations, IEEE transactions on pattern analysis and machine intelligence. 13(1991) 583-598.

[10]T. Y. Yuan, Z. G. Jiang, R. S. Meng, Automatic Splitting and Separating Overlapped Objects in Target-Segmented Image, Chinese Journal of Stereology and Image Analysis. 8(2003) 40-43. 